\title{
Editorial to the special issue on stochastics and probability in engineering mechanics
}

\author{
Christian Bucher $\cdot$ Antonina Pirrotta $\cdot$ Carsten Proppe $\cdot$ Luciano Rosati
}

Published online: 8 August 2019

(C) Springer Nature B.V. 2019

Probabilistic and statistical approaches to contemporary structural problems encountered in diverse technical disciplines is nowadays a crucial area of common interest to engineers and applied scientists belonging to different fields. For instance, recent examples in this area have dealt with structural systems subjected to random excitation, uncertainties related to model parameters, propagation of uncertainties on multiple scales, identification procedures and analysis of experimental data. Clearly, these problems often demand a high degree of sophistication in the numerical approaches and the development of innovative approaches for their solution is a challenging but, indeed, critical research topic. In this context, the objective of this special issue is to collect scientific contributions pertaining stochastic and probability-

C. Bucher

TU University of Vienna, Vienna, Austria

A. Pirrotta $(\bowtie)$

University of Palermo, Palermo, Italy

e-mail: antonina.pirrotta@unipa.it

A. Pirrotta

University of Liverpool, Liverpool, UK

C. Proppe

Karlsruhe Institute of Technology, Karlsruhe, Germany

L. Rosati

University of Naples, Naples, Italy based method in engineering mechanics, as well as presenting cross-disciplinary advances related to this topic. Further, the special issue of Meccanica entitled: Stochastics and Probability in Engineering Mechanics, comprises 13 papers, selected for their thematic richness and methodological diversity. The principle goal of this special issue is to encourage lively discussion, allowing it to serve as a forum promoting sustained and multidimensional activity in Stochastic Mechanics. The Guest Editors thank the Editor in chief: Professor Luigi Gambarotta, for this opportunity to publish the selected papers from authors belonging to the National and International Stochastic Mechanics Community and we would like to express our most heartfelt appreciation to each and every one of the authors, whose contributions are the cornerstone upon which the success of this special issue is built.

Publisher's Note Springer Nature remains neutral with regard to jurisdictional claims in published maps and institutional affiliations. 